\title{
Relacionamiento con los animales a partir de prácticas ecofeministas en el Eje Cafetero
}

\author{
Alexandra Giraldo Castro \\ alexa13.g@gmail.com \\ Universidad de Manizales \\ Stefany Gómez Murillo \\ stefany0190g@gmail.com \\ Universidad de Manizales
}

José Enver Ayala Zuluaga

jeayala@uniquindio.edu.com

\section{RESUMEN}

Profesor Asociado Universidad del Quindío

Este artículo es parte del estudio denominado: "Configuraciones que emergen de la relación desarrollo sostenible-ecofeminismo: un estudio regional", aquí se trata sobre el hecho de que los ecofeminismos son corrientes del feminismo que han planteado diversas formas de interacción con la vida humana y no humana. En ese sentido, desarrolla la propuesta de relacionamiento con los animales que ha surgido a partir de estas corrientes, en el territorio colombiano denominado "el Eje Cafetero". -En el trabajo de campo se identificó que surge una particular relación del desarrollo sostenible y el capitalismo, con la Naturaleza y los animales. Para analizar dicha relación se acudió a teorías latinoamericanas que han puesto en discusión el tema, al igual que a mujeres de la región con experiencias de vida de su diario quehacer; de igual manera, se consideraron Planes de Desarrollo municipales y departamentales, con el fin de identificar el abordaje en materia política a partir del feminismo y los animales. El artículo concluye con la identificación que, con base en diferentes postulados ecofeministas, el relacionamiento con los animales no humanos debe ser visto a partir de la ética y la empatía. Para las diferentes corrientes ecofeministas la lucha contra la opresión debe ser integral, inclusiva y abandonar la posición de opresión dominadora al utilizar los cuerpos de otros seres vivos, igualmente vulnerables, sintientes y explotados, de forma intensiva dentro de las prácticas del desarrollo sostenible, por lo que se acude a formas de vida como el veganismo y el consumo más consciente.

Palabras clave: Ecofeminismo; Desarrollo; Animales; Mujeres. 


\title{
Relationship with animals based on ecofeminist practices in the Coffee Region
}

\begin{abstract}
This article is part of the study called: "Configurations that emerge from the relationship sustainable development-ecofeminism: a regional study", here it is about the fact that ecofeminisms are currents of feminism that have raised various forms of interaction with human life and not human. In this sense, this article develops the proposal of relationship with animals that has emerged from these currents, specifically in the Colombian territory called "the Coffee Axis" in the field work, it was identified that a relationship of sustainable development and capitalism with Nature and animals. To analyze this relationship, Latin American theorists who have discussed the issue were used, as well as women from the region with life experiences from their daily work, in the same way, municipal and departmental Development Plans were considered, in order to to identify the policy approach to animals. The article concludes with the identification that, from different ecofeminist postulates, the relationship with nonhuman animals must be seen from ethics and empathy, because as Alicia Puleo indicates, the situation of non-human animals is the worst of all times since that share the planet with the human being. The fight against oppression must be comprehensive, inclusive and must abandon the position of oppressor and dominator by using the bodies of other equally vulnerable, sentient and exploited beings in an intensive way within the practices of sustainable development. of life as veganism and more conscious consumption.
\end{abstract}

Keywords: Ecofeminism; Development; Animals; Women.

Artículo recibido: 27 marzo 2021

Aceptado para publicación: 30 abril 2021

Correspondencia: alexa13.g@gmail.com

Conflictos de Interés: Ninguna que declarar 


\section{INTRODUCCIÓN}

Al generar discusiones sobre el relacionamiento con los animales a partir de prácticas ecofeministas en el Eje Cafetero, se encuentran diversos elementos convergentes, entre ellos, las responsabilidades directas que provienen del sistema capitalista y su concepto del desarrollo, así como de las ambigüedades del desarrollo sostenible, por ejemplo, los impacto sobre la Naturaleza y los animales de las prácticas que de allí se derivan. Las ecofeminismos en América Latina y por supuesto en Colombia, señalan crisis ecológicas y dominación de hombres sobre mujeres y de humanos sobre la Naturaleza, situaciones que responden a lo que el patriarcado ha legitimado al esencializar a las mujeres como lo Otro objetivado frente a lo plenamente humano, como la Alteridad (del latín, alter: otro), y a la Naturaleza como Vida Cíclica casi inconsciente (Wolf, 2001; Acero y Montenegro, 2019). En cambio, el Hombre (Varón) ha sido pensado como lo propiamente humano, como Razón, Mente, Espíritu y Cultura. (Puleo, 2019, p.26), generando la distancia y devaluación necesaria entre Hombre y Naturaleza.

Es pertinente recordar que el ecofeminismo es una corriente de pensamiento y praxis, que nace con la francesa Françoise D'Eaubonne (París, 1920-2005) en el año 1974, sugiriendo la unión o coincidencias entre el feminismo y la ecología. La pensadora libertaria basa el nexo entre los problemas ecológicos y de las mujeres, partiendo del momento en que la mujer fue considerada un terreno para poseer y fecundar por el hombre, al entender su papel en la procreación, convirtiendo a la mujer en un objeto apropiable, al mismo tiempo que iniciaba la ganadería y la anulación de formas de organización de las mujeres que les posibilitaban desarrollar cultivos agrícolas. Fertilidad y fecundidad se habrían convertido así en capacidades manejadas por los hombres. Surgen, en ese momento, las sociedades patriarcales que excluyen a las mujeres de las instancias de decisión. (Velasco, 2020; Duarte y García-Horta, y Puleo, 2011).

En el interior del desarrollo sostenible surgen proyectos que se basan en actividades de extracción de elementos naturales, como minería y petróleo, producción de biocombustibles y negocios monoproductivos de agricultura a gran escala, que arrasan con la diversidad ecológica de especies vegetales y animales, y conllevan al desplazamiento de comunidades, despojos y perturbaciones a los ecosistemas más 
frágiles, creando dependencias y forjando nuevas formas de dominación, especialmente sobre comunidades rurales campesinas, indígenas.

Ahora bien, Colombia, por supuesto, no es ajena a las diferentes problemáticas ambientales expresadas en los daños causados a la Naturaleza por las actividades de origen antropogénico basadas en la explotación intensiva de elementos naturales, o en la utilización de especies de fauna y flora para producción masiva. A lo largo del país, se extienden diversas prácticas que han causado deforestación, pérdida e interrupción de ecosistemas por causa de la ganadería extensiva (Mahecha, Zoot, Gallego y Peláez , 2002), por ejemplo, empresas multinacionales, especialmente, solicitan licencias para explotación de oro y cobre, como el caso de las solicitudes que han realizado a las autoridades ambientales en lugares cercanos al páramo de Santurbán en Norte de Santander (Ávila, 2020) ; la contaminación de los ríos por mercurio y cianuro resultante de actividades mineras que afecta poblaciones humanas, vegetales y animales; estas últimas también afectadas gravemente con la construcción de infraestructuras viales que responden a las exigencias del mercado para comunicar departamentos y municipios, facilitando la deforestación y pérdida de los hábitat de individuos de la fauna que, además mueren por la caza furtiva o atropellada por vías que interrumpen sus corredores naturales. Sin lugar a dudas, Colombia es una de las víctimas de los conflictos ecológicos que enfrenta América Latina, expoliada de los recursos naturales que atienden muchas de las necesidades de los países ricos con poder político y económico.

Lo expuesto anteriormente, deja entrever que en Colombia y el Eje Cafetero el liderazgo y visibilización de las mujeres en la defensa de la Naturaleza y en las luchas ambientales, son igual de significativas que en otros países de Latinoamérica. El hecho de que animalismo, ambientalismo, vegetarianismo, veganismo, feminismo, sean asumido como prácticas políticas por las mujeres en Colombia y el Eje Cafetero, permite evidenciar que se reconoce ya la histórica exclusión y sometimiento que conecta el patriarcado y el desarrollo impulsado por el capitalismo, que aun siendo planteado como sostenible, ha sido catalogado por varias autoras como mal desarrollo o insostenible (Santander y Mejía, 2015 y Rendón, 2007). El modelo de desarrollo vigente, basado en una razón meramente instrumental y de corto alcance, acarrea la destrucción del ecosistema global (Puleo, 2019, p.37). Resulta importante contextualizar 
acerca de los primeros acercamientos reconocidos entre las luchas por la liberación de las mujeres y las luchas por la liberación animal, en el instante en que ciertas feministas encontraron aspectos comunes en la dominación y opresión ejercida hacia las mujeres y los animales. Las primeras etapas del vínculo entre feminismo y defensa de los animales, corresponden al movimiento de mujeres sufragistas que se manifestaron en contra de la vivisección; “estas sufragistas fueron verdaderas pioneras del ecofeminismo al considerar a mujeres y animales como víctimas de la violencia patriarcal" (Puleo, 2019, p. 117).

En el eje cafetero de Colombia, proyectos de monocultivos, de construcción, plantaciones forestales cerca a fuentes hídricas, que generan contaminación de ríos y quebradas, así como de solicitudes para explotación de minería de oro y de otros metales y minerales, entre otros, han despertado sentires y necesidades de defensa y cuidado de la naturaleza, ante las aplanadoras políticas de entes territoriales y corporaciones regionales, que son permisivas y poco condicionantes con las empresas que llegan con intenciones de explotación de estos proyectos. Las situaciones de conflicto en municipios del eje cafetero tienen relación con el desarrollo y el crecimiento económico por medio de políticas de desarrollo urbano, como el caso de la ciudad de Manizales, en la que proyectos comunales de transformación conflictos socioambientales no sólo por un recurso físico, sino por la afectación al territorio de estas comunidades, que, más que un simple espacio significa su forma de vida (Munévar, 2017). Las mujeres de la región del eje cafetero también tienen un papel protagónico en las resistencias al lado de sus comunidades, sin embargo, a lo largo del país y en la región, esto está implicando una amenaza, pues las mujeres están siendo violentadas y asesinadas por defender los intereses legítimos de sus pueblos (Carrillo, Zapata \& Vázquez. 2009 y Timm, 2018)).

En dicho escenario, el resultado de tantas injusticias a las que se enfrentan Naturaleza, comunidades humanas vulnerables (mujeres, niños y niñas) y animales, plantean la imperiosa necesidad de pensarse a partir de otras perspectivas o epistemologías quizás experienciales) que cuestionen y reformulen las praxis actuales, en aras de dignificar las condiciones de vida futuras. Al respecto, el ecofeminismo es una redefinición de la realidad, como lo son el feminismo, el animalismo y el ecologismo, que se entrelazan en su teoría y práctica. (Puleo, 2019, p.15). En consecuencia, a partir de dicho prisma del 
feminismo, se desarrolló el estudio base de este artículo, y se generaron diálogos con mujeres de la región, para debatir entre las prácticas tradicionales del desarrollo sostenible, las alternativas al mismo y explorar el relacionamiento que florece, a partir del ecofeminismo, con los animales no humanos.

\section{ESTRATEGIAS METODOLÓGICAS, MATERIALES Y MÉTODOS}

Para el estudio se definió un enfoque cualitativo, pues el objetivo requería del entendimiento, la interpretación y compresión de las nuevas perspectivas y epistemologías nacientes, que varían de acuerdo a contextos económicos, sociales, políticos y ambientales, a partir del diálogo que se genera entre quienes realizaron la investigación y los y las sujetos cognoscentes, con los contenidos, sentidos y las mismas interpretaciones de las experiencias plasmadas de los diferentes sujetos de las fuentes consultadas, de acuerdo a sus realidades sociales, culturales y existenciales; haciendo uso para el estudio, de la investigación documental, acudiendo a lo propuesto por Galeano (2018), el cual se soporta en tres momentos; revisión de estudios previos, búsqueda y selección de información y, por último, hallazgo y comunicación de los resultados. Todo esto concordante con la explicación de Carlos Sandoval (1996) sobre la creación compartida del conocimiento que se genera mediante la interacción, “en la cual, los valores median o influyen la generación del conocimiento, lo que hace necesario "meterse en la realidad" objeto de análisis, para poder comprenderla tanto en su lógica interna como en su especificidad”'(p.29).

De igual manera, se realizó la selección de la unidad de trabajo en la población abordada, lo que dio lugar a contar con la participación de un grupo de mujeres (6) muy diverso en sus profesiones, pensamientos y oficios. Desde investigadoras, hasta activistas, campesinas y políticas que compartieron sus anécdotas y posturas frente a los ejes centrales del estudio.

Así mismo, se establecieron criterios de inclusión y exclusión delimitando la población y discursos, que permitieron interpretar las verdaderas manifestaciones de las relaciones de estudio en la investigación, utilizando fuentes primarias teóricas y políticas latinoamericanas, nacionales y locales, y se tuvieron en cuenta las indagaciones particulares sobre experiencias locales en los departamentos de Quindío, Risaralda y Caldas que se acercaban o manifestaban aspectos comunes sobre las relaciones analizadas. 
Las técnicas e instrumentos de recolección utilizados, así como las fuentes que nutrieron la información, fueron: la revisión documental de fuentes primarias como Planes de Desarrollo Departamental y Municipal de la región cafetera y sus respectivas ciudades capitales y las entrevistas en profundidad; como técnica de análisis se utilizó el análisis de contenido, no solo en función de las regularidades, también se asumieron las relevancias centrales en un proceso que se sistematizó a partir de las sábanas para su posterior triangulación, análisis e elaboración del proceso de conocimiento presentado.

\section{RESULTADOS Y DISCUSIÓN}

En el Eje Cafetero surgen feminismos y ecofeminismos que se expresan de formas diversificadas, configurando otros relacionamientos y perspectivas sobre el conjunto de la naturaleza. Al interior de esas transformaciones, también han reconocido que la perspectiva ética hacia la vida, debe incluir a los animales no humanos.

Dentro de esta investigación se analizó y denominó; relacionamiento con los animales, a los elementos encontrados en los Planes de Desarrollo de la región, en los cuales, se incluyen metas y programas conducidos a la atención de los animales y a aquellos testimonios que hicieron referencia a esos otros seres; como indica Puleo (2019); "junto al sexismo, al racismo, al clasismo y a otras formas de opresión por razón de orientación sexual o discapacidad, el "especismo" es una de las dominaciones que ha de considerar el ecofeminismo en busca de otro mundo posible"(p.103).

En primer lugar, es conveniente nombrar los primeros acercamientos reconocidos entre las luchas por la liberación de las mujeres y las luchas por la liberación animal, en el instante en que ciertas feministas encontraron aspectos comunes en la dominación y opresión ejercida hacia las mujeres y los animales. Las primeras etapas del vínculo entre feminismo y defensa de los animales, corresponden al movimiento de mujeres sufragistas que se manifestaron en contra de la vivisección. Finalizando los años mil ochocientos, las mujeres conectaron diferentes manifestaciones de maltrato hacia sus cuerpos y la manera en que estas eran ignoradas y observadas con indolencia, junto al sufrimiento al que eran sometidos los animales en los laboratorios. Estos movimientos de mujeres lograron que a mediados del siglo XIX, en países como Estados Unidos y Gran Bretaña, se comenzara a crear legislación en favor de los animales y la atención a través de refugios u hospitales veterinarios. Así mismo en el siglo XIX surgieron las primeras expresiones del ecofeminismo: 
[...] Las ecofeministas también sugieren que la conciencia que tienen las mujeres de la dominación patriarcal puede dar lugar a una actitud diferente hacia la naturaleza y los animales, en la que se rechazan las ideologías jerárquicas responsables de la subyugación tanto de las mujeres como de la naturaleza y se aboga por una relación no jerárquica e igualitaria entre ambos sexos, así como con la naturaleza y los animales. (Gonzales, $\mathrm{M}$ et al., 2008, p.91).

Lo anterior, hace parte de aquellos primeros enfoques de algunas corrientes ecofeministas, que aún están vigentes y que se han fortalecido mediante otras expresiones de activismo como el movimiento animalista o de la defensa de los animales: "a través del ecofeminismo, el feminismo puede redefinir a los animales humanos y no humanos y establecer nuevas formas de relación despojadas de explotación y violencia" (Puleo, 2019, p. 108). En ese sentido, dentro de los diálogos con las mujeres seleccionadas se expresaron asuntos como los siguiente;

[...] Tiene que ver con el nuevo caminar en las formas de relacionarnos entre los seres humanos, plantas, animales y libertades en el planeta, dándonos cuenta que tenemos que estar unidos no solo para informarnos, sino para apoyarnos y encontrar estrategias. (E2, A).

Las nuevas formas de comprender y situarse frente a la naturaleza, incluyen la compasión, la empatía por el sufrimiento al que se enfrentan los animales y el reconocimiento de sus capacidades per se; en ese sentido, se acude al enfoque de Nussbaum, al identificar que animales o criaturas sensibles son fines en sí mismas y deberían obtener un nivel mínimo de capacidades sobre determinados umbrales (Nussbaum, 2012), capacidades relacionadas por ejemplo con la sintiencia y su posibilidad de sentir dolor.

[...] la conducta respecto de los animales está ligada a la idea de «justica social», en la medida en que estos poseen la capacidad «sensitiva», «conativa»y «agencia». Es decir, el animal siente dolor, tiene una dimensión desiderativa y, sostengamos al menos de modo provisional, ejerce acciones más allá de su propia utilidad y bienestar. (Martinez, 2015, p.3). 
$\mathrm{Al}$ respecto, y partiendo de la empatía, emergen modos de vida como el veganismo, que contienen nexos con principios de corrientes ecofeministas. Para la definición del veganismo se pueden encontrar diversas propuestas; se puede entender como una posición ética y política que elimina de su vida cualquier producto de origen animal para la alimentación, ropa, cosméticos, y que rechaza la utilización y explotación de los animales, pues busca generar el menor daño posible a los otros animales con los que se habita el planeta. El veganismo acogido por el ecofeminismo con esa perspectiva "puede también leerse como una forma de resistencia a la colonización del cuerpo por parte de ese matrimonio también avenido que es el formado por el patriarcado y el capitalismo" (Albelda et al. 2018, p.221). La corriente ecofeminista crítica de Puleo llama a lo siguiente:

[...] El ecofeminismo demanda la reconciliación con los cuerpos y con su materialidad vulnerable. Recuerda que existe un amor sin odio, un deseo sin cosificación ni violencia. Al rechazar todo sistema de dominación, denunciando sus implicaciones patriarcales, el ecofeminismo llama a superar la violencia contra la naturaleza humana y no humana, así como los prejuicios antropocéntricos que legitiman la violencia contra los animales. (Puleo, 2019, p.127).

En las mujeres de la región estudiadas, se hallaron pruebas de la relación que también han introducido en su discurso y práctica, al hacer referencia a formas de vida como el vegetarianismo, veganismo y el cambio de visión utilitarista hacia los animales, transformando el relacionamiento con los mismos; la explotación la señalan, es ejercida a los ecosistemas y los animales. Sobre el asunto se encontraron manifestaciones, a continuación, expuestas:

[...] no puede uno ser ecofeminista y consumir derivados de la industria ganadera cárnica, la carme es lo que más huella hídrica tiene, es lo que más consume agua, están los gases de efecto invernadero, la producción de metano, la compactación del suelo, hay una pesca incidental, para sacar un kilo de pescado se tiene que sacar otros tantos kilos, es la compasión, esa empatía con los animalitos que son seres sintientes que sienten angustia, dolor, miedo, cariño y alegría. Por otro lado, el ambiental que son los animalitos, que son los principales de gases de 
efecto invernadero, de desforestaciones, de la propia infertilidad de los suelos y el tema político fuerte que es el paramilitarismo, con la ganadería es demasiado notoria, todo se entrelaza (E4, A.A).

Resulta interesante el reconocimiento de la capacidad de sentir de los animales, como también el hecho de la disminución o eliminación de productos animales, o la adopción de formas de vida como el veganismo, como respuesta también a dificultades ambientales que está generando la industria animal. Las nuevas formas de comprender y situarse frente a la naturaleza, incluyen la compasión y la empatía por el sufrimiento al que se enfrentan los animales (Mosterin, 2014 y Balza y Garrido, 2016).

Actividades como la ganadería, por ejemplo, están catalogadas, incluso por la Organización de las Naciones Unidas para la Alimentación y la Agricultura FAO, como uno de los mayores contaminantes y responsables de la emisión de gases de efecto invernadero y ha indicado también:

[...] El proceso de expansión de la ganadería que están viviendo los países de América Latina, representa tanto una oportunidad como una amenaza para el desarrollo sustentable de la región. Por un lado, es una oportunidad para generar riqueza y mitigar la pobreza si se toman las decisiones políticas adecuadas y se promueven sistemas de producción ganaderos sustentables y amigables con el ambiente. Por el otro, es una amenaza si la expansión de la actividad continúa sin considerar los costos ambientales y los potenciales efectos de marginalización de los pequeños productores. Esto puede incrementar los niveles de deforestación en la región, la degradación de los suelos, la pérdida de biodiversidad y la disminución del recurso hídrico, si no se toman medidas para evitarlo. Se deben tomar acciones decididas para que el crecimiento del sector se lleve a cabo de modo ambientalmente sostenible y que contribuya, al mismo tiempo, a la mitigación del cambio climático, de la pobreza y a la mejora de la salud humana. (FAO, sf).

No obstante, la ONU a través de la FAO no cuestiona el desarrollo en sí mismo que, al fomentar el crecimiento económico ha generado injusticia social, explotación animal y daño ecológico, principalmente en aquellos países catalogados como subdesarrollados o del tercer mundo, en los cuales ha transformado y moldeado las particularidades de las 
comunidades, cultural, social, económica y políticamente. Como lo menciona Escobar (2014):

[...] Puede decirse que el discurso del desarrollo ha operado a través de dos mecanismos principales: la profesionalización de "problemas de desarrollo", incluyendo el surgimiento de conocimientos especializados, así como vastas áreas del saber para lidiar con todos los aspectos del "subdesarrollo"; y, la institucionalización del desarrollo. (p. 29)

Para Escobar, estos mecanismos son elementos de un mismo universo discursivo sobre el desarrollo que se estableció como una potencia social efectiva y que se logra mediante la institucionalización del desarrollo con "una variada gama de organizaciones; desde las instituciones de Bretton Woods (Banco Mundial y Fondo Monetario Internacional) y otras organizaciones internacionales (sistema de la ONU), hasta las agencias nacionales y locales de planificación y desarrollo" (Escobar, 2014, p.29).

Sobre el desarrollo sostenible han surgido diferentes miradas y posiciones, por consiguiente es importante resaltar que, a pesar de que diferentes organizaciones multilaterales, entre esas, las Naciones Unidas, han reconocido que el crecimiento económico representa un elemento relevante en la crisis ambiental, las teorías frente a las transformaciones y transiciones que se forjan en espacios de discusión sobre el desarrollo, constituyen postulados superficiales al no basarlos en los criterios de producción y patrones de consumo imperantes del modelo económico actual que han generado desigualdades profundas y han arrojado a la naturaleza y a las diversas formas de vida humana y no humana a la crisis actual. Desde un punto de vista ecofeminista:

[...] La situación de los animales no humanos es la peor de todas las épocas desde que comparten el planeta con el ser humano. Por un lado, la crisis ecológica generada por el modelo devastador de desarrollo pone a la fauna silvestre al borde la extinción. Por otro, se ha construido un sistema monstruoso de campos de exterminio en que millones de animales destinados al consumo o la experimentación son privados de toda libertad y sometidos a atroces sufrimientos hasta la muerte. (Puleo, 2019, p.123). 
Al interior de corrientes ecofeministas se forja la interseccionalidad con la explotación de los animales, y en ellas se piensa para actuar y alcanzar justicia social para la naturaleza y los animales, abandonando posiciones androantropocéntricas. La defensa de los animales representa una evolución ética y moral. Una de las personas estudiadas indicó:

[...] yo fui vegetariana y luego vegana por el tema de la explotación. Si convivimos con otras formas de vida dejaremos de ver una relación utilitarista; como experiencia personal, estos temas me han cambiado la manera de relacionarme con los animales. $(\mathrm{E} 3, \mathrm{~A})$

Ahora bien, el movimiento animalista, en el que existe un importante liderazgo de mujeres, suma con su accionar a las discusiones políticas y legislativas en un país como Colombia, en el que se han suscitado debates en escenarios como el Congreso de la República, que han arrojado finalmente leyes de protección animal. En otros países, han surgido partidos políticos netamente animalistas que participan de decisiones gubernamentales, pero también de procesos de elección para cargos de representación ciudadana, como es el caso también de Colombia, donde en varios departamentos y municipios existen animalistas ocupando curules en corporaciones públicas. Los programas destinados a los animales a través de los Planes de Desarrollo, responden a disposiciones legales de diferentes normas nacionales, pero también corresponden a un asunto de voluntad política que resulta materializándose a su vez por el activismo y la participación de animalistas en la región, que propenden la inclusión de la protección y el bienestar animal en los instrumentos de planificación. Hay que aclarar que, en los planes de desarrollo, el bienestar se concibe específicamente para los animales domésticos o aquellos que conviven más directa y estrechamente con humanos, como perros, gatos, aves, conejos, entre otros, aunque a nivel nacional existan ciertas consideraciones de morigeración del dolor y del sufrimiento para los animales utilizados para consumo.

Se hallaron entonces metas destinadas a los animales en los Planes de Desarrollo; en el caso de Pereira (páginas 347, 348, 349, 443, 554,555), el municipio cuenta con una Política Pública reglamentada en el año 2018 y en la matriz estratégica del plan contiene acciones como la atención de casos de maltrato animal, esterilizaciones para el control poblacional, sistemas de identificación para los animales, centro de bienestar animal, 
campañas educativas sobre bienestar y tenencia responsable, entre otros. El municipio de Armenia (páginas 27, 28, 49, 66, 75), dentro de su Plan de Desarrollo estableció la creación de una oficina de bienestar animal, construcción de centro de bienestar animal, esterilizaciones para evitar la sobrepoblación de animales y, como Pereira, la elaboración de una Política Pública. Manizales (páginas 71, 72) como ciudad capital, Risaralda (páginas 106, 120, 220), Caldas (páginas 259, 260, 261, 262, 263) y Quindío (páginas 121, 125), también en sus Planes de Desarrollo incluyeron acciones enfocadas a la atención de la fauna doméstica. Mediante el activismo político (como una arista del animalismo) de personas pertenecientes al movimiento animalista se traduce, por medio de estos, la consigna de dar voz a los que no tienen voz.

En medio de esta discusión desarrollada entre el feminismo, ecologismo, ecofeminismo, veganismo y desarrollo sostenible, teniendo en cuenta aportes teóricos (Ko, 2019; Ruiz, 2016 y Gomez, 2012) y la valiosa contribución de personas objeto de este estudio de la región, se teje el deber ético que las mujeres del Eje Cafetero tienen que configurar otras relaciones con los animales no humanos, bien sea posicionadas en un movimiento feminista, ecofeminista o animalista, pues, se puede concluir que la lucha contra la opresión debe ser integral, inclusiva y debe abandonar la posición de opresora y dominadora al utilizar los cuerpos de otros seres igualmente vulnerables, sintientes y explotados de forma intensiva dentro de las prácticas del desarrollo sostenible, por lo que se acude a formas de vida como el veganismo y el consumo más consciente.

\section{CONSIDERACIONES FINALES}

Los animales como parte de la naturaleza también se constituyen víctimas de los modelos de desarrollo actual. Y frente a esto puede evidenciarse en los planes de desarrollo políticas que involucran estrategias de intervención y protección hacia los animales, bienestar y tenencia responsable que, aunque responden a disposiciones legales de diferentes normas nacionales, también corresponden a un asunto de voluntad política que resulta materializándose por el activismo y la participación del movimiento animalista en la región cafetera. El relacionamiento con animales no humanos, debe ser visto a partir de la ética y la empatía según postulados ecofeministas, y es un buen comienzo que, en la actualidad, algunas prácticas hacia las otras formas de vida estén involucradas en políticas públicas, pues tal como lo expresa Puleo, la situación de los animales no humanos es la peor de todas las épocas desde que comparten el planeta 
con el ser humano. (Puleo, 2019, p.123). Por ello la defensa de los animales representa una evolución ética y moral actual. A nivel político la defensa de los animales ha tomado gran relevancia y puede identificarse en la misma una importante participación femenina. La lucha contra la opresión debe ser integral, inclusiva y debe abandonar la posición de opresora y dominadora al utilizar los cuerpos de otros seres igualmente vulnerables, sintientes y explotados de forma intensiva dentro de las prácticas del desarrollo sostenible, por lo que se acude a formas de vida como el veganismo y el consumo más consciente.

Algunas reflexiones críticas se convierten en pensamientos colectivos y se materializan en prácticas comunitarias. Las mujeres se consideran víctimas de la crisis ambiental, pero también protagonistas del cambio hacia una nueva cultura ecológica no androcéntrica, y es allí como nacen otros conocimientos y saberes como la agroecología y el emprendimiento local en comunidades campesinas, los cuales eliminan la visión instrumental de la producción y de la tierra, y se cultiva, propendiendo el cuidado de la misma. Por otro lado, los conocimientos indígenas le apuntan al buen vivir, a la apropiación y defensa de sus territorios, bien sea por recuperarlos, conservarlos o fortalecer su arraigo, buscar autonomía, mantener y preservar su identidad.

El desarrollo sostenible busca justificar el crecimiento, dignificar la explotación de la naturaleza, poner los hombres y mujeres a su servicio, es un sistema de intercambio ascendente, que promueve el consumo no equitativo, y levemente inclusivo. El desarrollo en sí, no pone límites, y aunque bajo el calificativo de "sostenible" promovido por la ONU y aceptado por las economías capitalistas, pareciera ser más compasivo con la crisis ambiental, social y económica actual a través de sus Objetivos de Desarrollo Sostenible ODS, la realidad es que sigue siendo un favorecedor de la misma idea de desarrollo y no una alternativa al mismo.

Por su parte, el ecofeminismo es el nombre que recibe la combinación de todas las prácticas ecologistas liderada por mujeres en representación de otras mujeres, hombres, niños, niñas, campesinos y campesinas, comunidades indígenas, animales no humanos, entendiéndolas como un todo, sin distinción ni etiquetas. Es un movimiento que devela prácticas sensibles de orden ancestral, visibiliza otras economías y saberes a través del intercambio entre comunidades, rescata lo esencial y establece relaciones de cuidado que promueven una relación respetuosa con la naturaleza. Busca combatir el patriarcado 
sin minimizar al hombre, sino poniéndolo a la par de la mujer, fundamentados en principios de igualdad.

El ecofeminismo es diametralmente opuesto al desarrollo sostenible, considera en sí que la idea original de desarrollo fracasó, cuestiona los modelos de consumo y su propósito global pues, por el contrario, afirma que los grandes problemas se han acentuado, precisamente por sostener el desarrollo y no por buscar alternativas al mismo Es sano encontrar puntos en común entre ambos conceptos, a partir de lo que se refiere a la intención de cuidado del medio ambiente y la promoción de la igualdad de género, no obstante, el desarrollo sostenible y el ecofeminismo se abordan con lenguajes distintos, intereses opuestos y perspectivas que distan entre lo racional y lo sensible.

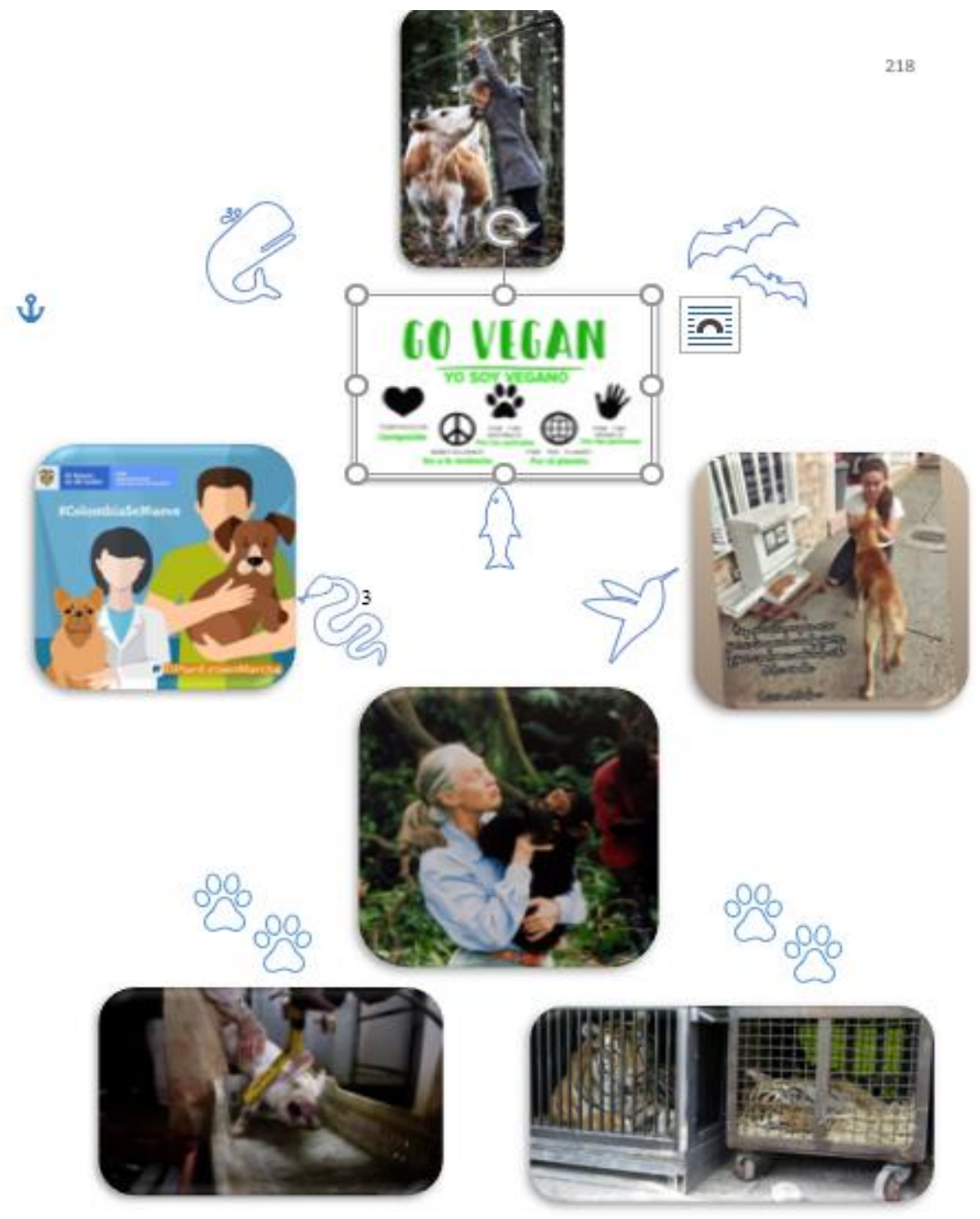




\section{LISTA DE REFERENCIAS}

Ávila, Ariel. (2019) detrás de la Guerra en Colombia. Bogotá: Planeta.

Acero Aguilar, Myriam; Montenegro Martínez, Leonardo (2019). La relación humano animal como construcción social. Tabula Rasa, 32, 11-16.

Albelda, J, Parreño, J, Marrero, J (2018). Humanidades ambientales. Pensamiento, arte y relatos para el siglo de la gran prueba. Madrid, España. Los libros de la Catarata.

Balza, I. \&. (2016). ¿Son las mujeres más sensibles a los derechos de los animales? Sobre los vínculos entre el animalismo y el feminismo. Filosofía moral y política, 289-305.

Carrillo Franco, Blanca Estela, Zapata Martelo, Emma, \& Vázquez García, Verónica. (2009). Violencia de género hacia mujeres del Frente de Pueblos en Defensa de la Tierra. Política y cultura, (32), 127-147. Recuperado en 07 de marzo de 2021, de $\quad$ http://www.scielo.org.mx/scielo.php?script=sci_arttext\&pid=S0188$\underline{77422009000200007 \& \operatorname{lng}=\mathrm{es} \& \ln \mathrm{ln}=\mathrm{es}}$.

Escobar, A (2014). Sentipensar con la tierra Nuevas lecturas sobre desarrollo, territorio y diferencia. Medellín Colombia. Ediciones Unaula.

Galeano, Maria E. (2018) Estrategias de investigación social cualitativa- El giro en la mirada. Medellín Colombia. Universidad de Antioquia

Gómez, L. (2012). El Ecofeminismo de Donna J. Haraway Gestión y Ambiente, pp. 165-206 Universidad Nacional de Colombia Medellín, Colombia

Gonzales, M, y Rodríguez, J (2008). Al margen de los márgenes: encuentros y desencuentros entre feminismo y defensa de los animales. Razonar y actuar en defensa de los animales. (pp. 83-106). Madrid, España: Los libros de la Catarata.

Ko, Aph (2019). "Cinco razones por las que los derechos de los animales son una cuestión feminista", El Salto. Recuperado de https://www.elsaltodiario.com/antiespecismo/cinco-razones-por-las-que-losderechos-animales-son-una-cuestion-feminista- 
Mahecha, L; Zoot, M; Gallego, Luis y Peláez, F. (2002) Situación actual de la ganadería de carne en Colombia y alternativas para impulsar su competitividad y sostenibilidad. En: Rev Col Cienc Pec. 213-225.

Martinez, P. (2015). El «enfoque de las capacidades» de Martha Nussbaum frente el problema de la ética animal. Veritas no.33 Valparaíso. Recuperado de: https://scielo.conicyt.cl/scielo.php?script=sci_arttext\&pid=S071892732015000200004

Mosterín, J, 82014). La compasión por los animales. El triunfo de la compasión. Nuestra relación con los otros animales. Alianza: Madrid.354 p.

Munévar, C., Dávila, C. y Giraldo, R. (2017). Conflictos en contextos socioambientales en la eco-región eje cafetero. Cuatro casos paradigmáticos. de los conflictos socioambientales a las resistencias territoriales. 25-68. http://ceanj.cinde.org.co/programa/archivos/publicaciones/colección_virtual/8_d e_los_conflictos_ambientales.pdf

Nussbaum, M. (2012). El enfoque de las capacidades. Propuesta para el desarrollo humano. Barcelona: Paidós. Recuperado de: https://scielo.conicyt.cl/scielo.php?script=sci_arttext\&pid=S071892732015000200004

Organización de las Naciones Unidas para la Alimentación y la Agricultura. (sf). Oficina Regional de la FAO para América Latina y el Caribe. Ganadería sostenible y cambio climático en América Latina y el Caribe. Recuperado de:http://www.fao.org/americas/prioridades/ganaderia-sostenible/es/

Puleo, A. (2011). Ecofeminismo para otro mundo posible. Madrid, España. Ediciones Cátedra (Grupo Anaya. S.A.)

Puleo, A. (2019). Claves Ecofeministas para rebeldes que aman a la tierra y a los animales. Madrid, España. Plaza y Valdés Editores.

Sandoval, C. (1996). Investigación cualitativa. Bogotá Colombia. instituto colombiano para el fomento de la educación superior, icfes. 
Santander, L., \& Mejía, L. y. (2015). El desarrollo sustentable y la instrumentalización de su paradigma. En E. S. February, Special edition develonment (págs. 97-103). Mexico: Moderna.

Rendón Acevedo, J. A. (2007). El desarrollo humano sostenible: ¿un concepto para las transformaciones? Equidad y Desarrollo, (7), 111-129.

Ruíz, María (2016). "Feminismo y antiespecismo: dos luchas con mucho en común". Recuperado de https://www.diagonalperiodico.net/libertades/29659-veganismoy-lucha-social.html

Timm, A. K. (2018). Feminicidio extractivista. Reflexiones sobre la violencia hacia las mujeres defensoras del agua y los territorios. En A. J. Erpel, Mujeres en defensa de territorios reflexiones feministas frente al extrativismo (págs. 91-101). Santiago: Cono Sur.

Velasco, a. (7 de 03 de 2021). Nueva Sociedad. Obtenido de Ética animal y feminismo: hacia una cultura de paz.

Wolf, U. (2001).Leben mit Tieren. Die Hauptformen und ihre ethischen Implikationen“ en ZDF-Nachtstudio, ed., Mensch und Tier, Geschichte einer heiklen Beziehung, Frankfurt, 40-59. 


\section{ILUSTRACIÓN}

Arbelaez, C. (2020). (foto). Recuperado de:

https://www.facebook.com/AnimalesCalarca/photos/a.891019081100525/14475 01148785646

Biography. (S.F). How Jane Goodall Became One of America's Most Notable Scientists Without a College Degreehttps://www.biography.com/news/jane-goodallchimpanzees-college-degree. (figura). Recuperado de https://www.biography.com/news/jane-goodall-chimpanzees-college-degree

Código Nuevo. (2018). Por qué somos conscientes del horror de los mataderos pero seguimos comiendo carne. (figura). Recuperado de https://www.codigonuevo.com/conciencia-social/conscientes-horror-mataderosdejamos-carne

Cuerpomente. (2020). Mujeres que luchan por los animales. ¡Conócelas!. (Figura). Recuperado de: https://www.cuerpomente.com/ecologia/estilos-devida/unbound-project-mujeres-activistas-derechos-animales_2190.

Departamento de Planeación Nacional. Recuperado de: https://pbs.twimg.com/media/EAAV_LTXYAATPXV.jpg

Duarte Cruz, J. M. y García-Horta, J. B. (2016). Igualdad, Equidad de Género y Feminismo, una mirada histórica a la conquista de los derechos de las mujeres. Revista CS, no. 18, pp. 107-158. Cali, Colombia: Facultad de Derecho y Ciencias Sociales, Universidad Icesi. doi: 10.18046/recs.i18.1960

Pet5stars. (sf). Sí yo soy vegano. (figura). Recuperado de: http://www.pet5stars.es/index.php/en/noticias/61-si-yo-soy-vegano

Recuperado de: http://1.https//www.kinderworld.org/es/videos/industria-decarne/matadero-vacas/ 